\title{
Why do Patients with Acute Pneumonia Receive Intravenous Infusions?
}

Igor Klepikov MD

Professor, retired, USA.

*Corresponding Author: Igor Klepikov, Professor, retired, USA.

Received date: September 22, 2021; Accepted date: October 13, 2021; Published date: October 21, 2021

Citation: I Klepikov. (2021). Why do Patients with Acute Pneumonia Receive Intravenous Infusions?. Biomedical Research and Clinical Reviews. 5(1) DOI: 10.31579/2692-9406/085

Copyright: (C2021 Igor Klepikov. This is an open-access article distributed under the terms of the Creative Commons Attribution License, which permits unrestricted use, distribution, and reproduction in any medium, provided the original author and source are credited.

\begin{abstract}
:
Currently, almost all urgently hospitalized patients immediately get access to the venous bed and begin to receive an infusion of solutions. This priority of this procedure is due not only to the need to have the most effective way of administering medications, but also to compensate for the loss of fluid, which in acute diseases has many reasons for this. Further recommendations for the correction of water-electrolyte and volume losses and the choice of the infusion rate are determined by the general criteria for their diagnosis in accordance with the parameters of the large circle of blood circulation. Considering AP, first of all, as a result of infection and not focusing on the localization of the process, modern medicine does not make exceptions in this therapeutic direction for patients with inflammation of the lung tissue.
\end{abstract}

Keywords: pneumonia; infusions; diagnosis; abdominal cavity; dogmas; synchronicity; acute disease

\section{Introduction:}

Currently, almost all urgently hospitalized patients immediately get access to the venous bed and begin to receive an infusion of solutions. This priority of this procedure is due not only to the need to have the most effective way of administering medications, but also to compensate for the loss of fluid, which in acute diseases has many reasons for this. Further recommendations for the correction of water-electrolyte and volume losses and the choice of the infusion rate are determined by the general criteria for their diagnosis in accordance with the parameters of the large circle of blood circulation. Considering AP, first of all, as a result of infection and not focusing on the localization of the process, modern medicine does not make exceptions in this therapeutic direction for patients with inflammation of the lung tissue.

For many years, fever and tachypnea were considered the main causes of fluid deficiency in patients with AP [1]. But the role of these factors in the occurrence of inconspicuous losses is hardly worthy of comparison with the consequences of homeostasis disorders accompanying such diseases as, for example, enterocolitis or peritonitis, when the body really loses large volumes of fluid. Such losses are very noticeable and can be estimated both quantitatively and qualitatively.

Try to compare the volume and quality of losses through the gastrointestinal tract that occur as a result of repeated vomiting and diarrhea, as well as intense exudate into the intestinal lumen and into the abdominal cavity, with the evaporation of fluid during breathing and an increase in body temperature. Even if we imagine that all patients with AP during hospitalization in the hospital have a respiratory rate twice higher than normal, and the body temperature is stable at 39 degrees Celsius, the approximate loss of fluid from the body as a result of increased evaporation will not be able to approach the volume and quality of the above emissions. Nevertheless, all patients with a severe and aggressive course of the disease, regardless of the diagnosis, are recommended to inject fluid with boluses [2-5].

The differences between obvious and hidden fluid losses in patients with inflammatory processes should inevitably raise doubts that the main cause of circulatory disorders in AP is dehydration as a result of increased sweating. As is known, such hemodynamic disorders can occur at the very beginning of inflammation of the lung tissue, sometimes literally within a few hours. The unlikely role of evaporation mechanisms in the development of severe circulatory disorders in AP gave reason to interpret such aggressive forms of the disease as sepsis and septic shock [2, 4, 5].

In the literature, information about the frequency of detection of bacteria in the blood of patients with AP does not receive attention. It can be found only in isolated reports, in which the release of the pathogen from the blood of patients with this disease is only from $2-9 \%$ to $12 \%$, including not only cases of sepsis and septic shock, but also bacteremia [6-8]. Analyzing septic complications of various diseases, some authors draw attention to the fact that bacteriological blood testing in patients with AP usually does not give results $[9,10]$. Even in patients with AP and septic shock, the frequency of positive blood cultures is only from $13.2 \%$ to $18 \%$, while the comparative mortality rates from this complication with positive and negative tests do not have significant differences [11, 12]. 
Declaring the penetration of the pathogen into the bloodstream as the cause of severe AP does not mean that the diagnosis of septic complications corresponds to reality. However, the concept of the leading role of the pathogen in the clinical manifestations of the process requires analysis and interpretation, and such explanations are beginning to be associated with the effect of preliminary antibacterial therapy $[9,13,14]$. The paradoxical nature of this conclusion is obvious, since it turns out that, on the one hand, antibiotics effectively destroy bacteria, but, on the other hand, they are not able to prevent the development of septic complications. This strange and illogical explanation is just an attempt to bring incomprehensible facts into line with familiar dogmas.

And yet, despite the lack of objective arguments in favor of the alleged causes of hemodynamic disorders in patients with AP, such factors undoubtedly exist and manifest themselves quite quickly and hard. The use of various methods for assessing the severity of the condition of these patients shows a characteristic feature of clinical manifestations with a tendency to develop hypotension. This feature is noted with the help of widely used diagnostic methods, which include a set of different tests: APACHE II (Acute physiology and chronic health assessment II), CURB65 (confusion, urea, respiratory rate, blood pressure, age $\geqslant 65$ years), PSI, the method of determining which is not much different from the first method [15-18].

A common feature of these methods, which are popular in intensive care units, for determining the severity of the condition and the prognosis of patients is that they do not reflect differences in diagnoses and localization of the main process. In addition, one of the most important tests in all severe patients is the indicator of peripheral blood pressure. This generalization of various diseases and the interpretation of disorders in accordance with the same pathogenesis scheme is the main drawback when evaluating patients with AP.

In order to understand the reason for the distorted assessment of the condition of patients with different localization of the primary focus of inflammation and to find out the origins of the signs that force intravenous infusions in AP, it is necessary to recall some fundamental canons of medicine and well-known facts.

Firstly, inflammation of the lung tissue remains the only disease among such processes that occurs in the small circle of blood circulation.

Secondly, the synchronicity of the work of the two halves of the cardiovascular system is ensured by the entry of equal volumes of blood into them and significantly lower pressure in the pulmonary vessels.

Thirdly, the violation of parity between the small and large circles of blood circulation is incompatible with life, and functional deviations that constantly occur both in the process of vital activity of the body and in the case of diseases are leveled by the autonomous regulatory system.

Fourth, the inflammatory transformation of tissues is based on a sequential step-by-step reaction of blood vessels in the affected area with a violation of blood flow and a sharp increase in the permeability of the vascular wall.

Finally, an acute inflammatory process is inevitably accompanied by five classic signs described many centuries ago by Celsus and Galen and received indisputable confirmation on the basis of subsequent medical experience: heat, pain, redness, swelling, and loss of function. Therefore, these factors will act independently of our attitude to them, and the violation of function will determine the features of the clinic of the disease, depending on the affected organ.

If we now take into account the presented information, the functional consequences of inflammation in the small circle of blood circulation begin to radically differ from similar processes of peripheral localization. The following diagram can give a general idea of the significance of the localization of the focus of inflammation for the blood circulation of the body (see Figure 1, Comment 1). This image is a clear proof of the fact that a sudden lesion of the lung tissue creates a physical barrier to the general blood flow, while other localizations are accompanied by a violation of blood circulation in the regional segments of the vascular network. 


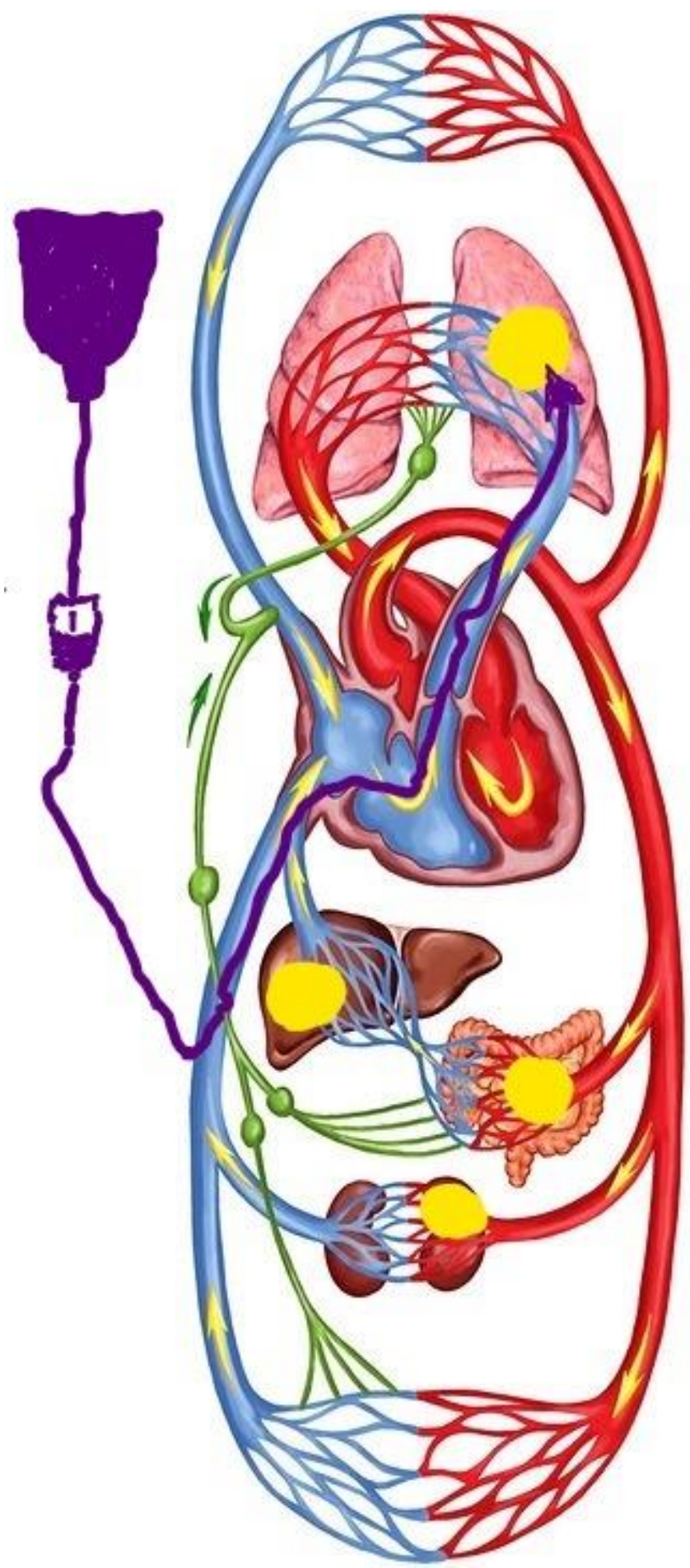

Figure 1: Schematic representation of the human circulatory system.

1. The comparative value of foci of acute inflammation (yellow fields) for different departments and volumes of blood flow, depending on the possible localization.

2. The initial route of intravenous administration of solutions (dark purple arrow).

However, the influence of the focus of inflammation in the lung on the blood flow in the small circle of blood circulation is much more complex and broader than just a physical obstacle, depending on the volume of tissue damage. The regulation of the life balance between the two circulatory circles works independently of our desires with the slightest shifts and obstacles. For almost a century, it has been known about the presence of baroreceptors in the pulmonary vessels, the irritation of which is accompanied by their spasm and a decrease in pressure in the peripheral vessels of the large circle [19]. This reflex mechanism, known in the medical literature as the discharge reflex, is our salvation in the event of unforeseen circumstances that have arisen in the vascular pool of the lungs. 
This adaptive mechanism, despite its protective nature, in the case of rapid development of pathological processes can lead to situations that themselves require immediate elimination. But now the direction of medical care is determined by the interpretation of the reasons that in this case need to be eliminated, and the result of these efforts will depend on the influence of a particular technique on the pathogenetic mechanisms of the disease.

Since hypotension in patients with AP is currently considered as a manifestation of sepsis and septic shock (see above), intravenous bolus infusions are recommended in such situations by analogy with extrapulmonary causes of septic complications [2-5]. At the same time, up to $40-50 \%$ of the total mass of septic complications are patients with $\mathrm{AP}$, in which a different mechanism of circulatory disorders is the leading one [4].

The mechanism of hypotension in the large circle of blood circulation in $\mathrm{AP}$ as a result of damage to the pulmonary vessels was presented above, and this sign of the disease is especially manifested in the aggressive development of the process. In this situation, it is not difficult to imagine the role played by infusions that increase venous return and additional blood flow to the focus of inflammation (Fig. 2, comment 2). However, the effect of infusion therapy on the dynamics of AP is also a much more complex process than its visual version shown in the figure.
The need to clarify the role of infusion therapy in the dynamics of the development of lung inflammation arose in our work many years ago, when the most aggressive bacterial forms of AP began to be purposefully hospitalized in the our department during the initial period of the disease. The concentration of a large number of such patients was accompanied by the rapid development of pleural complications and high mortality in them. A typical example of such a development of events is our following observation.

A 2-year-old girl was taken to the clinic with abdominal pain and shortness of breath 12 hours after their appearance. According to her medical history, the child was healthy, but in the last few days she had a mild respiratory syndrome with a runny nose and a cough without fever. Upon admission to the clinic, the patient was diagnosed with AP (Fig. 2). Intensive treatment was immediately started, including intravenous administration of two antibiotics and intravenous fluids up to $30 \mathrm{ml} / \mathrm{kg} /$ hour for 2 hours, followed by a decrease in the infusion rate to $10 \mathrm{ml} / \mathrm{kg} /$ hour. Despite the treatment, the child's condition did not improve, and a control radiograph was diagnosed with pyopneumothorax 36 hours after hospitalization (Fig. 3). The pus obtained from the pleural cavity during drainage was subjected to bacteriological and microscopic examination, but no microflora was found in it.

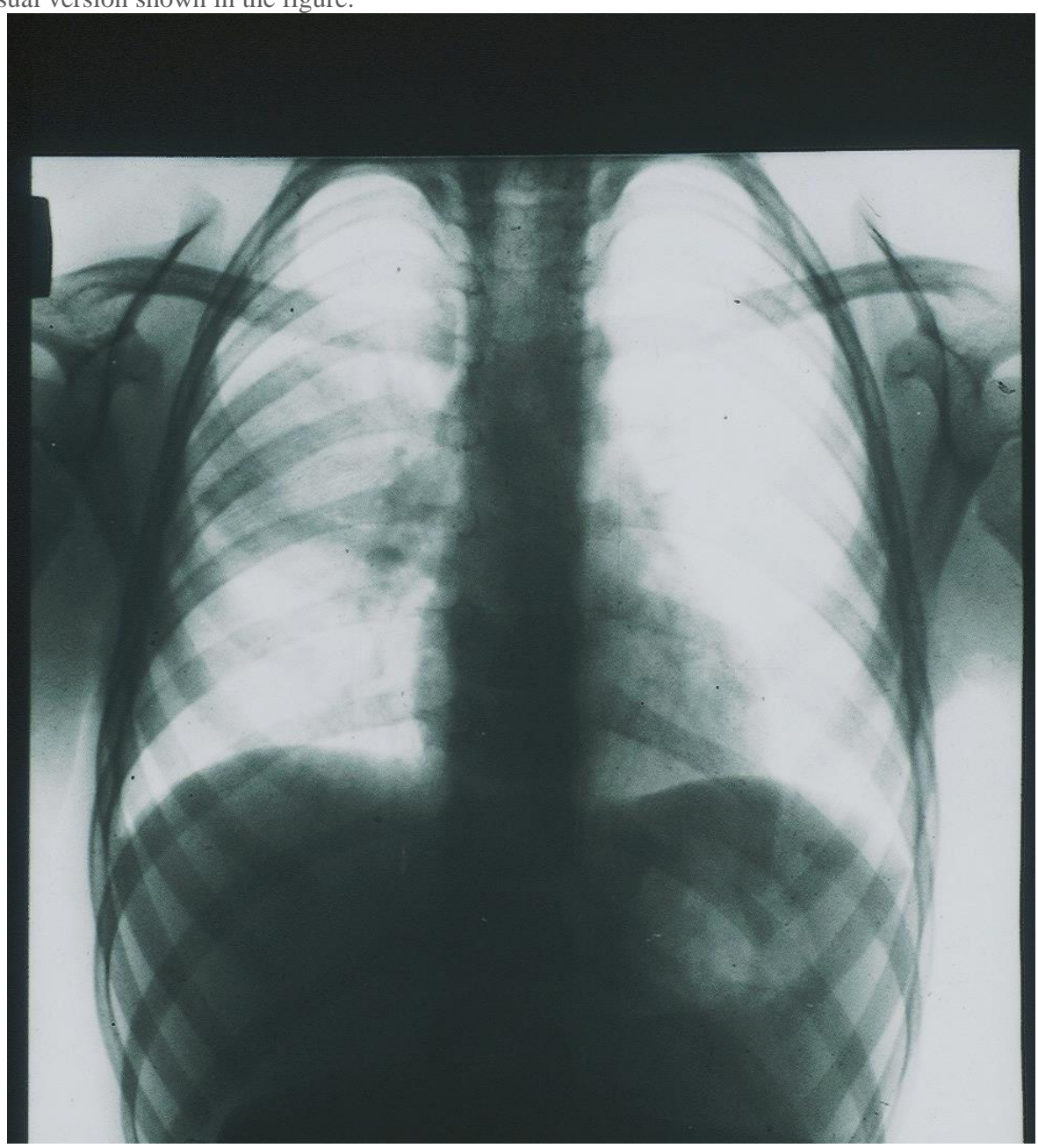

Figure 2: X-ray photograph of 2 y.o. girl 12 hours after the first signs of AP with abdominal pain syndrome were discovered. There is homogeneous shading in a middle-right pulmonary field. 


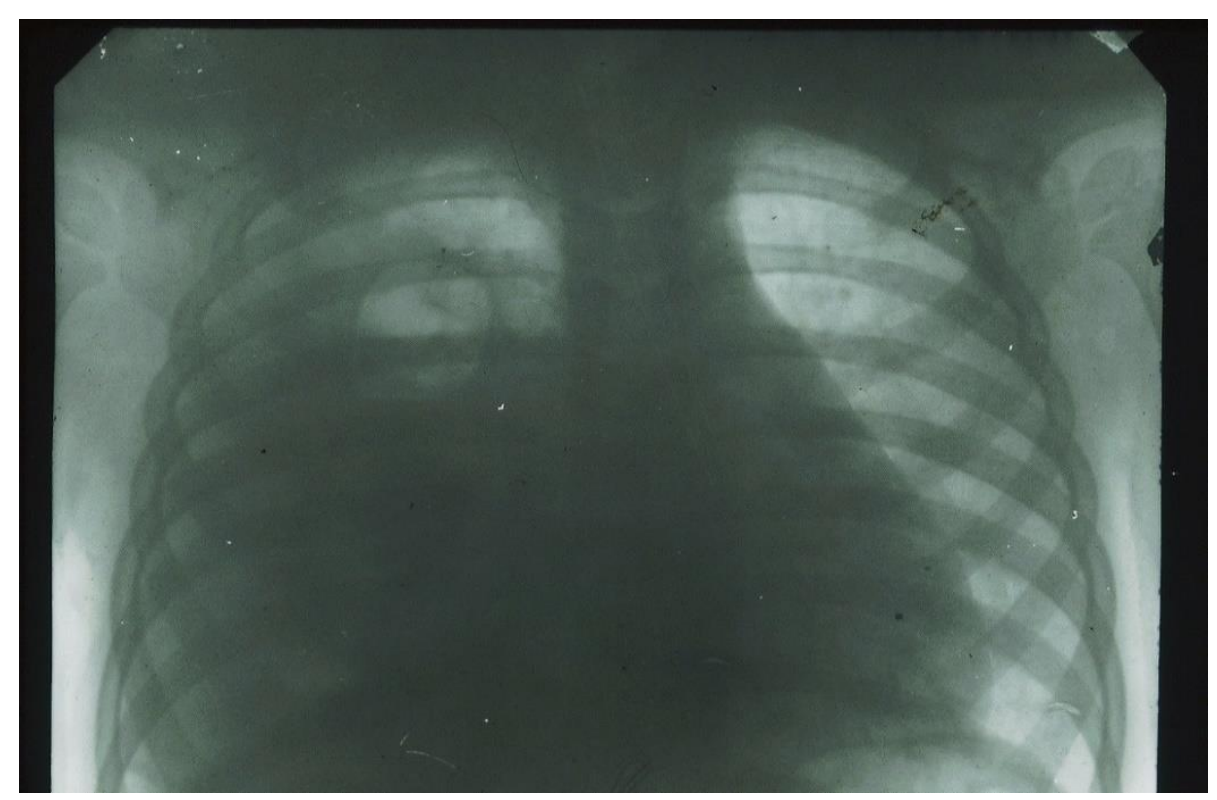

Figure 3. X-ray of the same patient (see Fig. 3), 36 hours after the start of inpatient treatment.

There is an intense uniform darkening of almost the entire right hemithorax with a displacement of the mediastinum to the left, as well as a cavity with a fluid level in the upper pulmonary field.

The presented observation cannot be an absolute proof of the negative effect of infusion therapy on the dynamics of the inflammatory process in the lung. The results of the observed transformation in the area of inflammation only allow us to assume such a dependence and draw appropriate conclusions on an empirical basis. Therefore, in order to find additional arguments in favor of such an assumption, which cannot be obtained in clinical conditions, animal experiments were conducted.

The volume of the description of experimental studies does not allow us to present them in the framework of a journal article. However, if it is necessary to obtain this information, it can be found in available sources $[20,21]$. Only the section of the study that is directly relevant to the issue under discussion is given here.

First of all, a model of the bronchogenic form of AP was created. At the same time, in order to reduce the charismatic etiology of the disease and to assess the significance of other factors, cultures of microbes that are usually not considered as pathogens of AP were used. The choice was made in favor of Escherichia coli and Staphylococcus epidermidis. When a statistically reliable stable production of the AP model was obtained, in the final series of experiments, intravenous infusions of solutions were administered to rabbits during the occurrence of inflammation in the lungs. The volume of infusions was $30 \mathrm{ml} / \mathrm{kg} /$ hour and was performed once a day for 3 days. In addition, in 6 cases, the addition of a methylene blue dye to the infusion solution was used. This technique was borrowed from the experiments of V. Menkin, who discovered the permeability factor [22].

The results obtained after euthanasia of animals on the fourth day of the experiment showed the following. Reproduction of the AP model without subsequent intravenous infusions was accompanied by the development of local inflammation of the lung tissue with a slight pleural reaction in some cases. Intravenous infusions in all cases were accompanied by the development of parapneumonic pleurisy. In two cases, pyopneumothorax was detected, the cause of which was small foci of destruction in the lung tissue. After infusions with the addition of dye, weakly colored lung tissues were found along the periphery of the inflammatory focus (Fig.4).

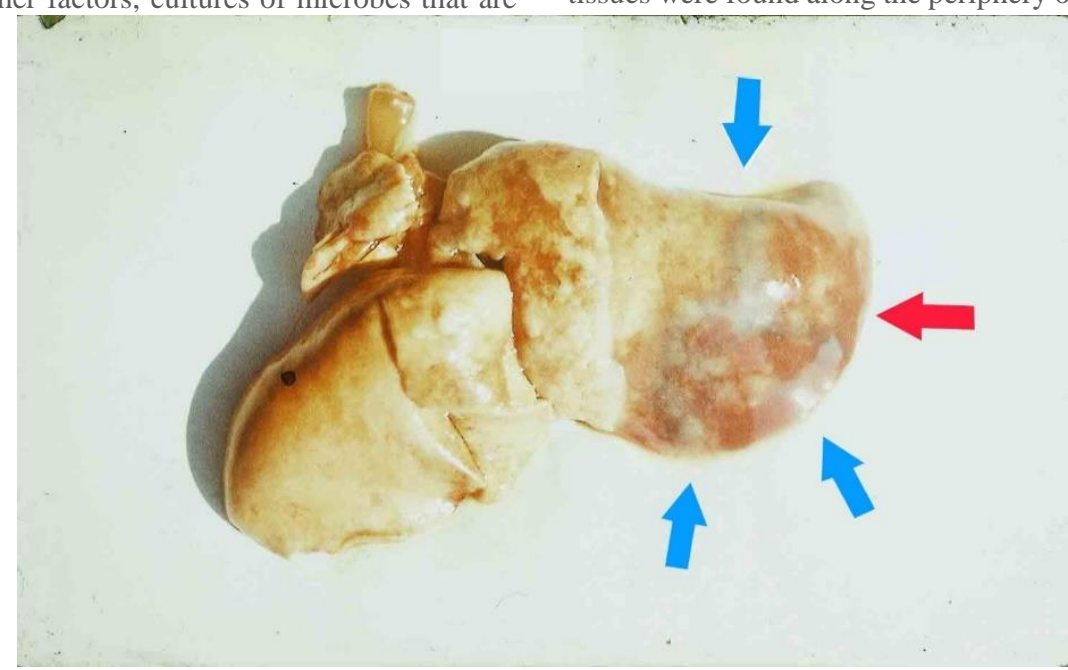

Figure 4: Macro-preparation of the lung (experiment, series 4b). Massive focus of the inflammation in a pulmonary surface (red arrow), surrounded by the additional sections of infiltration with blue shading (blue arrows). Explanations in the text. 
Thus, the results of the experiments allowed us to obtain additional and undoubted confirmation of the negative role of intravenous infusions in AP. The use of the dye demonstrated a visual effect of the spread of inflammatory infiltration in the lung tissue, which is a consequence of an increase in blood flow to the area with increased vascular permeability. In addition, it should be emphasized that, despite the strict repetition of the experimental conditions in each specific case, the final results were distinguished by individual diversity.

The totality of the facts presented above gave grounds to exclude any intravenous infusions in patients with AP, especially in the initial period of the disease. As an alternative to first aid for such patients, instead of infusion therapy, methods were used to relieve reflex spasm of the vessels of the small circle and at the same time reduce venous return (cupping therapy, body cooling, vagosympathetic blockade). The results of such a transformation of medical care in aggressive forms of AP exceeded our expectations [20, 21].

Unfortunately, the results of this work for a number of reasons were not presented in a timely manner in the most widespread English-language scientific literature. Today, the previous stereotypes of treating this category of patients with intravenous infusions continue to dominate in wide world practice, and the final materials indicate a continuing increase in pleural empyema in patients with AP, even in regions with developed health systems [23,24].

The additional information presented above gives an idea of one of the pathogenetic mechanisms of stimulating the inflammatory process in the lungs. Such an analysis allows us to understand why the frequency of parapneumonic effusion in community-acquired pneumonia reaches 20$50 \%$ of cases, and the empyema of the pleura can be sterile in microbiological studies [25].

The development of the SARS-CoV-2 pandemic has not changed the strategy for solving the problem of lung tissue inflammation. The causative agent of the process and its suppression continue to be the main goal of current research and therapeutic efforts. The mechanisms of the disease development are studied mainly at the cellular and molecular level. The dependence of AP manifestations on known biological rules, compensatory reactions of the body, their manifestation and the level of permissible deviations do not receive due attention in discussions and search works. After all, like A.H. Attaway and co-authors rightly point out [26], modern respiratory care revolves around auxiliary measures.

Meanwhile, the appearance of new facts only confirms the materials already passed over the past decades, in which the change in the pathogens of AP did not affect its leading characteristics. The pathogenetic mechanisms of viral pneumonia have the same basis as the bacterial forms. For example, the use of tomography in patients with COVID-19 pneumonia to determine the volume of blood in the lungs allowed us to establish a decrease in this indicator in small vessels with a diameter of no more than $2 \mathrm{~mm}$ [27]. The authors suggest that this fact is caused by spasm and thrombosis of small vessels as a result of their viral damage. Generalized thrombosis of this localization can hardly be compatible with life, but the spasm of the pulmonary vessels fully corresponds to the already known and mentioned above discharge reflex.

Thus, modern therapeutic efforts aimed at correcting systemic circulatory disorders are the result of an incorrect interpretation of their causes and mechanisms. The long-term fascination with the etiology of AP and the etiotropic principles of treatment has left a deep didactic trace with the oblivion of many biological laws. Bringing the concept of the disease in line with the basics of medical science should be the first stage of further revision of the complex of therapeutic measures.

\section{References}

1. D. A. Berlin, R. M. Gulick, F. J. Martinez. (2020). Severe Covid-19. December 17, 2020. N Engl J Med. 383:2451-2460.

2. Rhodes A, Evans LE, et al. (2017). Surviving Sepsis Campaign: International Guidelines for Management of Sepsis and Septic Shock. Intensive Care Medicine. 43 (3): 304-377.

3. Lim TK, Chew MY. (2018). Management of severe community acquired pneumonia in the emergency department. J Emerg Crit Care Med. 2:2.

4. Ceccato A. Torres. (2018). Sepsis and community-acquired pneumonia. Ann Re Hosp. 2:7.

5. Alhazzani W et al. (2020). Surviving sepsis campaign: Guidelines on the management of critically ill adults with coronavirus disease 2019 (COVID-19). Crit Care Med.

6. J. P. Metlay, G. W. Waterer, A. C. Long at al. (2019). on behalf of the American Thoracic Society and Infectious Diseases Society of America. "Diagnosis and Treatment of Adults with Community-acquired Pneumonia. An Official Clinical Practice Guideline of the American Thoracic Society and Infectious Diseases Society of America". American Journal of Respiratory and Critical Care Medicine. 200(7); 45-67.

7. Sanjay Sethi (2020). Community-Acquired Pneumonia. Merck Manual for the Professional.

8. G. W. Waterer, M. W. Quasney, R. M. Cantor, R. G. Wunderink. (2001). Septic Shock and Respiratory Failure in Community-acquired Pneumonia Have Different TNF Polymorphism Associations. AJRCCM. 163(7).

9. Lin GL, McGinley JP, Drysdale SB et al. (2018). Epidemiology and immune pathogenesis of viral sepsis. Front Immunol, 9:2147.

10. Weiss S L, Peters M J, Alhazzani W. et al. (2020). Surviving sepsis campaign international guidelines for the management of septic shock and sepsis-associated organ dysfunction in children. Intensive Care Med. 46; 10-67.

11. Garcia Vidal C, Ardanuy C, Tubau F, et al. (2010). Pneumococcal pneumonia presenting with septic shock: hostand pathogen-related factors and outcomes. Thorax. 65:77-81.

12. Hadil A. Al Otair, Mohammed A. Hussein, Mohamed A. Elhoseny et al. (2015). Severe pneumonia requiring ICU admission: Revisited. Journal of Taibah University Medical Sciences. 10(3); 293-299.

13. Liapikou A, Ferrer M, Polverino E et al. (2009). Severe Community-Acquired Pneumonia: Validation of the Infectious Diseases Society of America/American Thoracic Society Guidelines to Predict an Intensive Care Unit Admission. Clin Infect Dis. 48: 377-385.

14. Restrepo MI, Mortensen EM, Rello J et al. (2010). Late admission to the ICU in patients with community-acquired pneumonia is associated with higher mortality. Chest. 137: 552557.

15. Knaus WA, Draper EA, Wagner DP, Zimmerman JE. (1985). APACHE II: a severity of disease classification system. Crit Care Med. 13(10):818-829.

16. Murillo-Zamora, E, Medina-Gonzalez, A, Zamora-Perez, L, et al. (2018). Performance of the PSI and CURB-65 scoring systems in predicting 30-day mortality in healthcare-associated pneumonia. Med Clin (Barc) 150: 99-103.

17. Ilg, A, Moskowitz, A, Konanki, V, et al. (2019). Performance of the CURB-65 score in predicting critical care interventions in patients admitted with community-acquired pneumonia. Ann Emerg Med 74: 60-68. 
18. Junnian Chen, Bang Liu, Houwei Du et al. (2021). Performance of CURB-65, PSI, and APACHE-II for predicting COVID-19 pneumonia severity and mortality. European Journal of Inflammation. 19.

19. Schwiegk, H. (1935). Der Lungenentlastungsreflex. Pflügers Arch. ges. Physiol. 236, 206-219.

20. Klepikov I. (2020). Acute pneumonia. New doctrine and first treatment results. Lambert Academic.

21. Igor Klepikov (2021). How many Pneumonias exist in nature? Generis Publishing.

22. Menkin Valy (1940). "Dinamics of inflammation". Macmillan.

23. Arnold D, Hamilton F, Morris T, et al. (2019). S12 The changes in incidence and management of pleural empyema in England over the last decade. Thorax. 74:9-10.
24. Bobbio A, Bouam S, Frenkiel J, et al. (2021). Epidemiology and prognostic factors of pleural empyema. Thorax.

25. Hassan M, Patel S, Sadaka A S, Bedawi E O, Corcoran J P, \& Porcel J M. (2021). Recent Insights into the Management of Pleural Infection. International journal of general medicine. 14; 3415-3429.

26. Attaway A H, Scheraga R G, Bhimraj A. (2021). Severe covid19 pneumonia: pathogenesis and clinical management. BMJ.

27. Thillai M, Patvardhan C, Swietlik EM, et al. (2021). Functional respiratory imaging identifies redistribution of pulmonary blood flow in patients with COVID-19. Thorax. 76:182-184.
This work is licensed under Creative Commons Attribution 4.0 License

To Submit Your Article Click Here: Submit Manuscript

DOI: $10.31579 / 2692-9406 / 085$
Ready to submit your research? Choose Auctores and benefit from:

$>$ fast, convenient online submission

$>$ rigorous peer review by experienced research in your field

$>$ rapid publication on acceptance

$>$ authors retain copyrights

$>$ unique DOI for all articles

$>$ immediate, unrestricted online access

At Auctores, research is always in progress.

Learn more auctoresonline.org/journals/biomedical-research-andclinical-reviews- 